\title{
Magnetic resonance imaging of endocardial exits from epicardial ventricular tachycardia substrates in left ventricular nonischemic cardiomyopathy
}

\author{
Christopher Reithmann ${ }^{1}$, Bernhard Herkommer ${ }^{1}$, Theresia Kling ${ }^{1}$, Cordula Brauns ${ }^{1}$, \\ Michael Fiek ${ }^{1}$, and Michael Ulbrich ${ }^{1}$ \\ ${ }^{1}$ Helios Clinic Munich West
}

April 3, 2021

\begin{abstract}
Introduction: In patients with left ventricular (LV) nonischemic cardiomyopathy and monomorphic ventricular tachycardia (VT), midmyocardial and epicardial substrates are often involved but endocardial structures may also be affected. Delayed enhancement - magnetic resonance imaging (DE-MRI) was used to characterize the substrates of predominantly epicardial VT to improve identification of target sites for ablation. Methods and Results: 12 patients with LV nonischemic cardiomyopathy and monomorphic VT (prior myocarditis in 9) had a predominantly epicardial $(\mathrm{n}=8)$ or epicardial-only DE-MRI substrate $(\mathrm{n}=4)$. Modest-sized endocardial involvement in predominantly epicardial substrates was identified by DE-MRI in 8 patients. Mapping of 22 VTs was performed in 12 patients using an endo-epicardial approach in 6 patients and an endocardial-only approach in 6 patients. Endocardial VT reentry circuit exit sites as defined by entrainment and pace mapping criteria corresponded to endocardial breakthroughs from predominantly epicardial DE-MRI substrates in 7 patients. The endocardial VT exits were located at the ventricular base near the mitral annulus in 6 patients. Successful endocardial ablation of at least one VT was accomplished in 5 patients. Epicardial ablation as a part of an endo-epicardial approach or as epicardial-only ablation was performed in 6 patients and was successful in 4 patients. Conclusion: Endocardial breakthroughs from predominantly epicardial DE-MRI substrates are often located near the ventricular base in the perivalvular region and correlate with endocardial VT reentry circuit exit sites amenable to ablation.
\end{abstract}

\section{Hosted file}

breakthroughs MRI.pdf available at https://authorea.com/users/405652/articles/516567magnetic-resonance-imaging-of-endocardial-exits-from-epicardial-ventricular-tachycardiasubstrates-in-left-ventricular-nonischemic-cardiomyopathy 

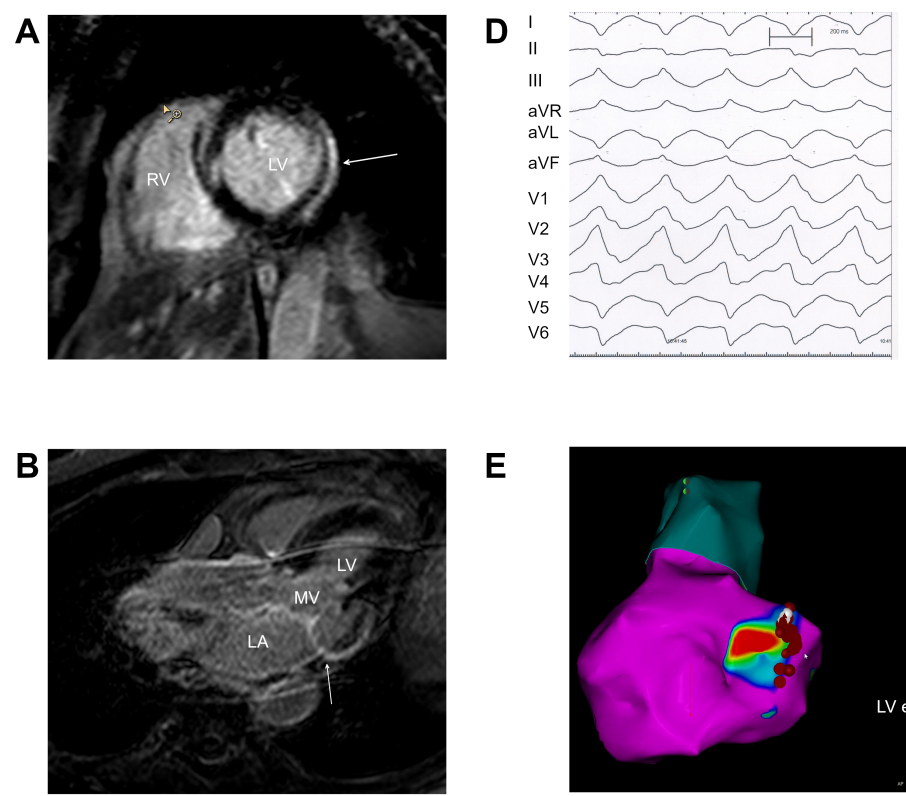

E
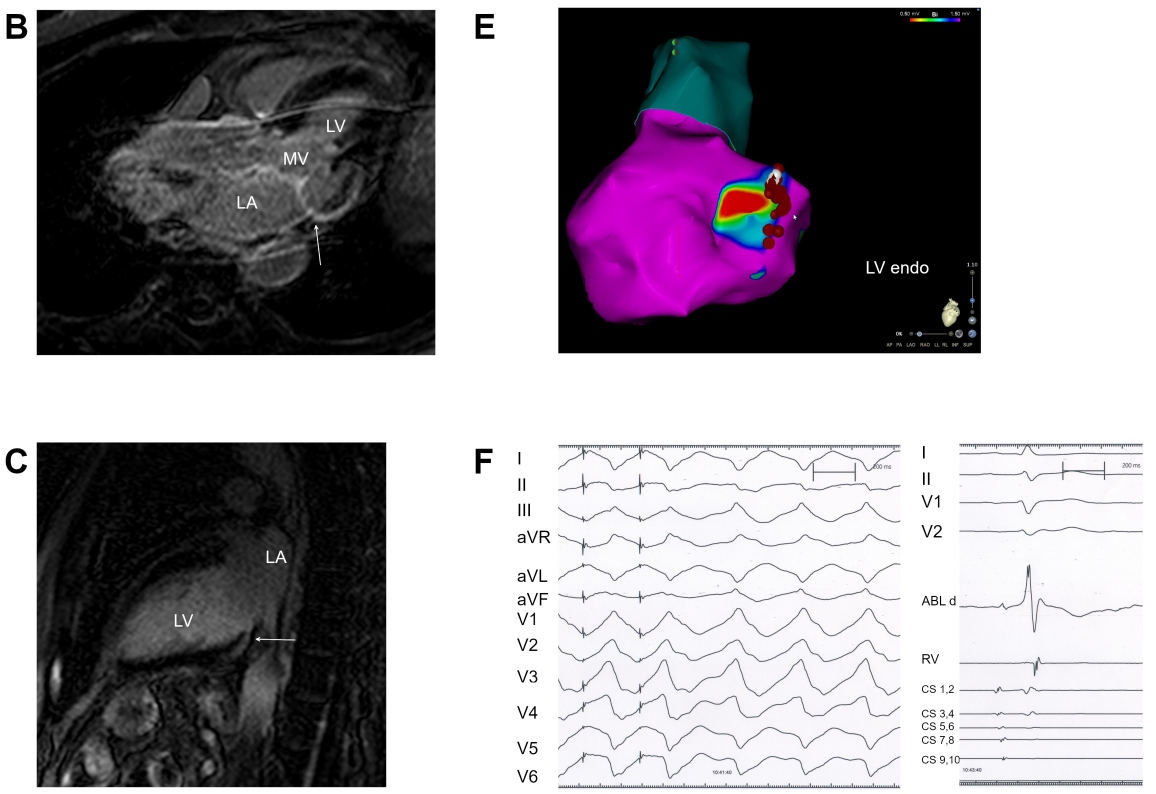
G
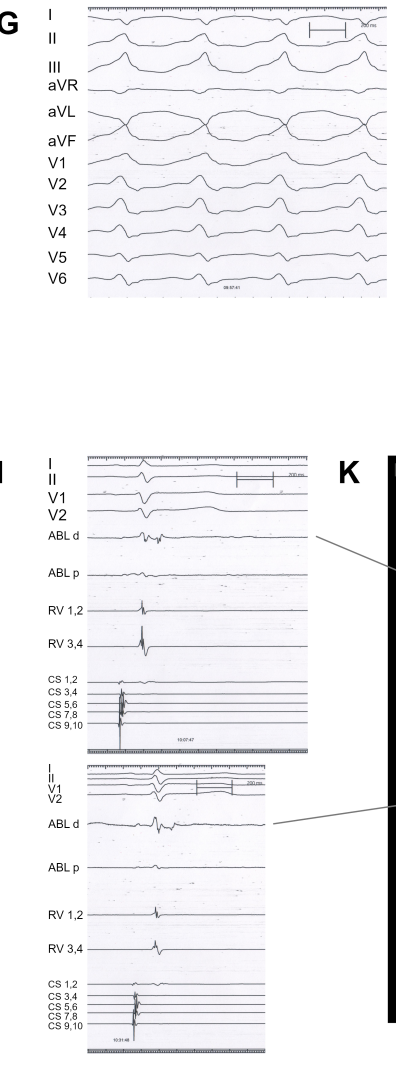

A

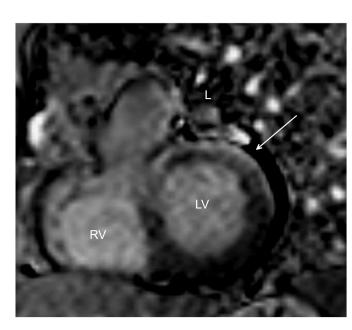

B

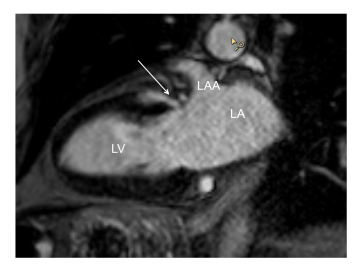

H
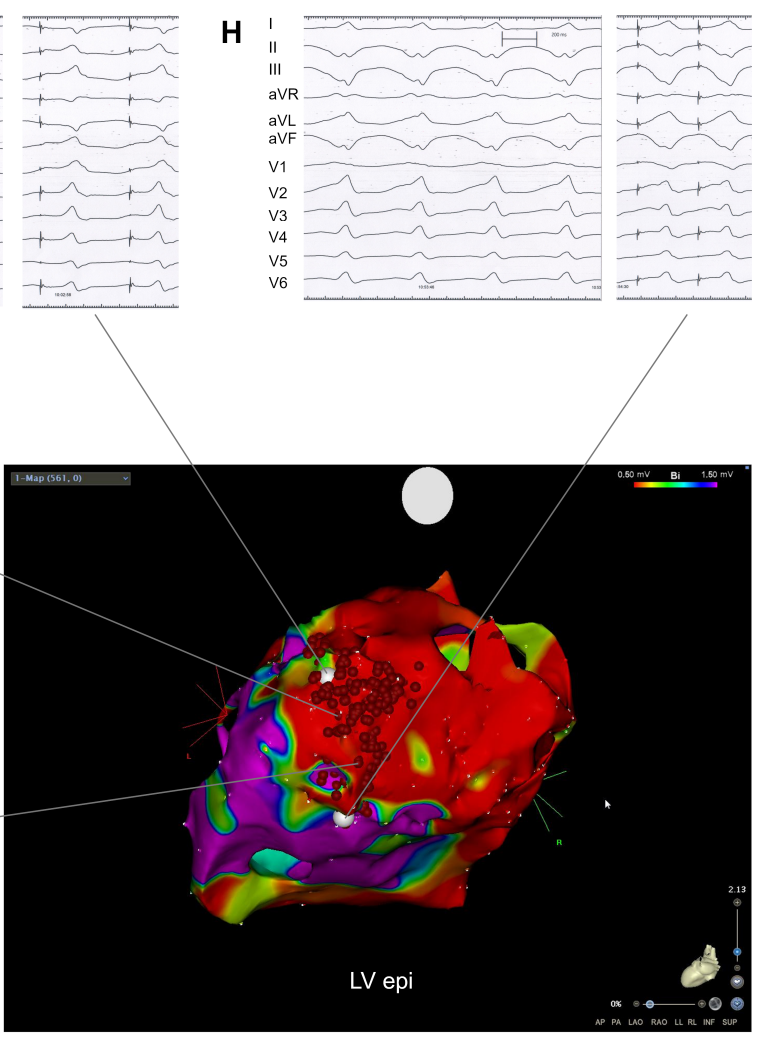

C

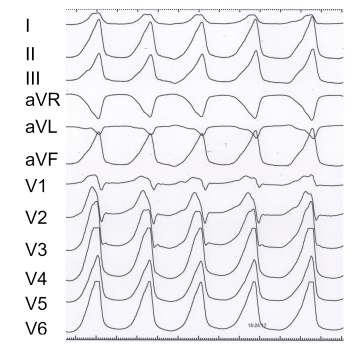

D

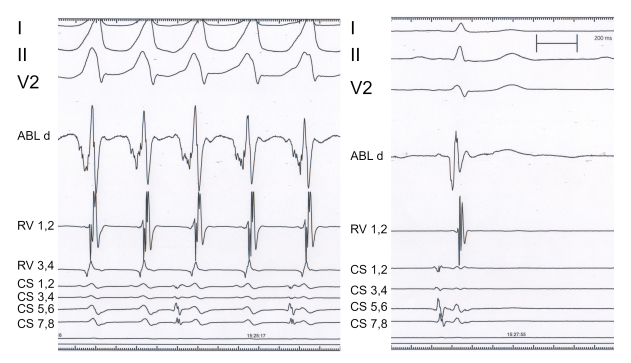


A

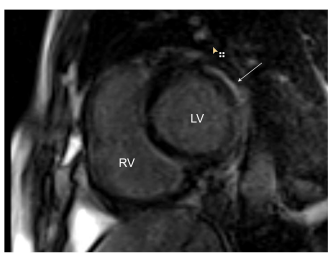

B

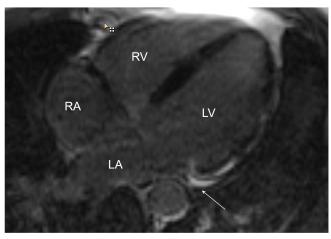

C

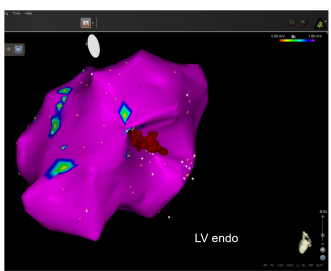

D

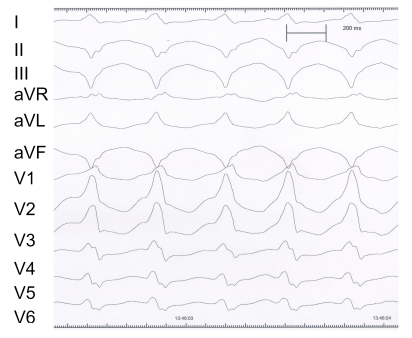

E

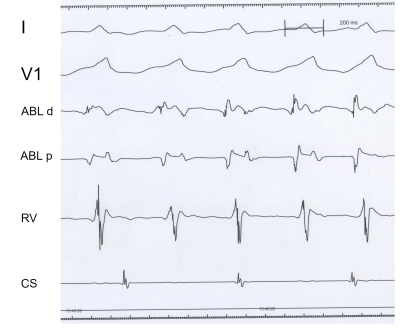

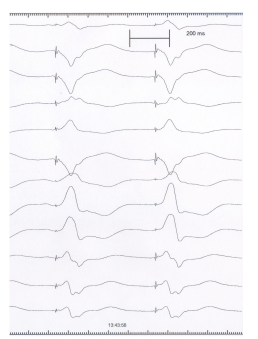


A

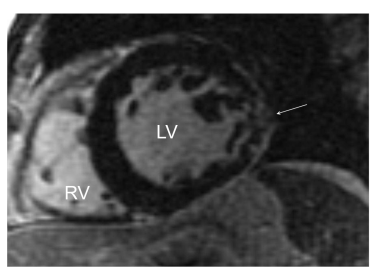

B

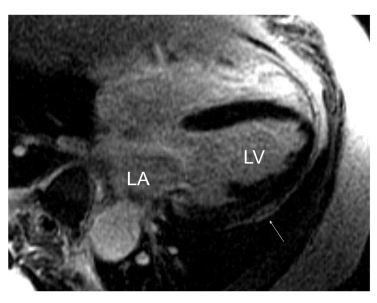

D

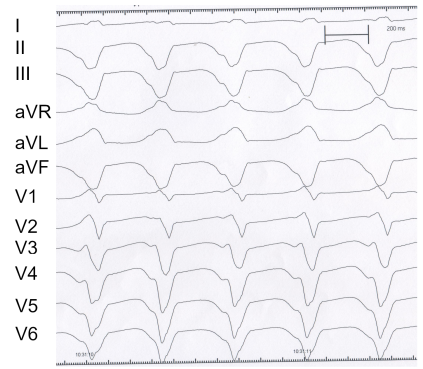

E

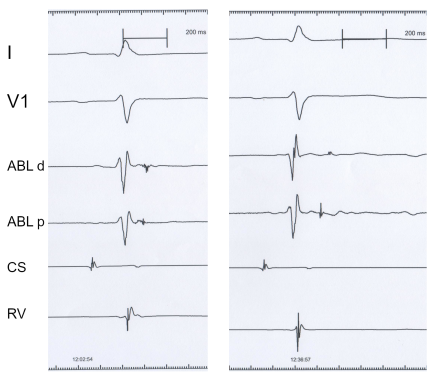

$\mathbf{F}$

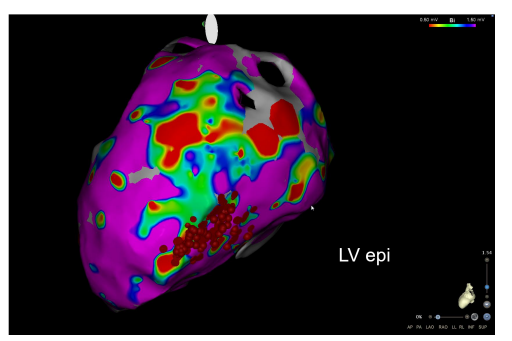

\section{Hosted file}

breakthroughs table 1.pdf available at https://authorea.com/users/405652/articles/516567magnetic-resonance-imaging-of-endocardial-exits-from-epicardial-ventricular-tachycardiasubstrates-in-left-ventricular-nonischemic-cardiomyopathy 